\title{
13. Ethischer Diskurs zu Epigenetik und Genomeditierung: die Gefahr eines (epi-)genetischen Determinismus und naturwissenschaftlich strittiger Grundannahmen $^{1}$
}

\subsection{Einführung}

Das folgende Kapitel soll einen Einblick in die vielfältigen ethischen Debatten geben, die im Zusammenhang mit Genetik und Epigenetik geführt werden. Besonders kontrovers diskutiert wird darunter momentan das Thema Keimbahneingriffe durch Genomeditierung an menschlichen Embryonen. Begriffe wie "Genomeditierung“, „CRISPR/Cas“2 oder „Designerbaby“ sind zu Schlagworten geworden. Die Vorstellung, durch Genomeditierung ein „Kind nach Wunsch zu designen“, ist allerdings eine äußerst verzerrte Wiedergabe der tatsächlichen Möglichkeiten, die durch neue gentechnische Verfahren im therapeutischen Bereich eröffnet werden (siehe Fehse et al., Kap. 9). Diese sind wesentlich begrenzter als es der in diesem Zusammenhang problematische Begriff des „Designens“ annehmen lässt. Denn um ein solches „Kind nach Wunsch“ durch Veränderung des Genoms „zu designen“, wäre es zum einen erforderlich, dass durch

1 Dieses Kapitel basiert auf Arbeit aus dem philosophischen Teilprojekt des Projekts „COMParative ASSessment of Genome- and Epigenome-Editing in Medicine: Ethical, Legal and Social Implications/Vergleichende Bewertung des Einsatzes der Genom- und Epigenom-Editierung in der Medizin: Ethische, rechtliche und soziale Implikationen“ (COMPASS-ELSI), gefördert durch die Deutsche Forschungsgemeinschaft (DFG - 409799774).

2 CRISPR: „Clustered Regularly Interspaced Short Palindromic Repeats“; Cas: „CRISPR associated enzymes“. CRISPR/Cas wird in der Gentherapie eingesetzt, um gezielt an DNA zu binden und diese ggf. zu verändern. Es handelt sich hierbei um ein gentechnisches Verfahren, dessen Ursprung in einer Art bakterieller Immunabwehr liegt. 
CRISPR/Cas und andere Verfahren der Gentechnik jedes beliebige Gen geändert werden könnte, was die gentechnologischen Möglichkeiten allerdings übersteigt. Zum anderen müssten wir davon ausgehen, dass eine solche genetische Veränderung ausreicht, um jede beliebige Eigenschaft in einem zukünftigen Kind herbeizuführen. Letzteres setzt die Annahme eines genetischen Determinismus ${ }^{3}$ voraus. Der Einsatz des CRISPR/ Cas-Verfahrens innerhalb der Gentherapie kommt allerdings nicht nur auf Keimbahnebene in Betracht. Vielmehr ergeben sich mit der neuen Technik zukünftig vermutlich auch Möglichkeiten, die DNA-Sequenz von Körperzellen (somatische Genomeditierung, siehe Fehse, Kap. 6) oder das Epigenom, d. h. die räumliche Struktur der DNA, ohne Veränderung der Basenabfolge (Epigenomeditierung, siehe Walter/Gasparoni, Kap. 3) zu verändern. Während bei der Genomeditierung die DNA-Sequenz geändert wird, ist es Ziel der Epigenomeditierung, z. B. durch Veränderung des Methylierungszustandes der DNA oder des Acetylierungszustandes der Histone ${ }^{4}$ die epigenetische Konfiguration von Chromatin zu modifizieren (siehe Tab. 1).

Tabelle 1: Genomeditierung und Epigenomeditierung

\begin{tabular}{|c|c|c|}
\hline & Genomeditierung & Epigenomeditierung \\
\hline Verfahren & $\begin{array}{l}\text { Änderung der DNA-Sequenz } \\
\text { (Basenabfolge): } \\
\text { - von Keimbahnzellen, } \\
\text { z. B. bei Embryonen (Keimbahneingriff) } \\
\text { - oder von Körperzellen (somatische } \\
\text { Genomeditierung) }\end{array}$ & $\begin{array}{l}\text { Änderung der DNA-Struktur zur Beein- } \\
\text { flussung der Transkription der DNA in der } \\
\text { Zelle („Ablesen“) und der Genexpression } \\
\text { (Umwandlung der DNA in Proteine): } \\
\text { - i. d. R. von Körperzellen (somatische } \\
\text { Epigenomeditierung) }\end{array}$ \\
\hline Anwendungsfälle & $\begin{array}{l}\text { Therapie genetisch bedingter Erkrankungen } \\
\text { - z. B. Hämoglobinopathien } \\
\text { ( } \beta \text {-Thalassämie, Sichelzellanämie, ...) } \\
\text { Was sind Hämoglobinopathien? } \\
\text { - Störung der Bildung oder Funktion des } \\
\text { roten Blutfarbstoffs Hämoglobin mit z. T. } \\
\text { schwerwiegender Symptomatik }\end{array}$ & $\begin{array}{l}\text { Therapie epigenetisch bedingter } \\
\text { Erkrankungen } \\
\text { - z. B. Imprinting-Disorders } \\
\text { (Prader-Willi-Syndrom, Angelman- } \\
\text { Syndrom, ...) } \\
\text { Was sind Imprinting-Disorders? } \\
\text { - durch fehlerhaftes „Ablesen“ von Genen } \\
\text { bedingte Syndrome mit einer Kombination } \\
\text { aus neurologischer Symptomatik, Wachs- } \\
\text { tums- und Stoffwechselstörungen }\end{array}$ \\
\hline
\end{tabular}

Naturwissenschaftlicher Funktionszusammenhang:

- Genom- und Epigenomeditierung: Methoden der Gentechnologie

- Wie bei der Genomeditierung kommt auch bei der Epigenomeditierung neben Zinkfingernukleasen (ZFN) und „Transcription Activator-Like Effector Nucleases" (TALENs) häufig der "CRISPR/Cas-Komplex" zum Einsatz.

3 Die verschiedenen Begriffe (hier auch -ismen) werden im folgenden Text erläutert, für eine Übersicht siehe auch Tab. 2.

4 Histone sind Proteine, um die der DNA-Doppelstrang gewunden ist. 
- Dieser wird jedoch so verändert, dass das Cas-Enzym, das bei der Genomeditierung i. d. R. eingesetzt wird, um einen DNA-Bruch herbeizuführen, als sogenanntes "catalytically deactivated-Cas" (dCas) vorliegt.

- Durch den Einsatz von "CRISPR/dCas" bei der Epigenomeditierung wird somit eine Bindung an die DNA ermöglicht, ohne dass es zu einem DNA-Bruch kommt.

Auch Fragen der Epigenetik wurden in der Vergangenheit nicht nur in der Wissenschaft, ${ }^{5}$ sondern auch in Populärwissenschaft ${ }^{6}$ und Ethik in den Fokus genommen und geben Anlass zu ganz unterschiedlichen Vorstellungen von Vererbung und Verantwortung. Hierbei werden allerdings oft verzerrte naturwissenschaftliche Vorstellungen zugrunde gelegt. In der ethischen Analyse der Epigenetik wird zudem mitunter darauf verwiesen, dass sich in Debatten zur Epigenetik ein verdeckter Gendeterminismus (Schuol, 2016 und 2017) in Form eines epigenetischen Determinismus (Waggoner/Uller, 2015) fortsetzt. Weder genetischer noch epigenetischer Determinismus sind jedoch aus naturwissenschaftlicher Sicht bestätigt. Um einen kritischen Umgang mit wissenschaftlichen Erkenntnissen und ethischen Argumenten im Zusammenhang mit Genomeditierung und Epigenetik zu ermöglichen, ist es daher wichtig, zu verstehen, welche Konzepte, z. B. zum Einfluss von DNA auf unser Leben, in Debatten rund um Genomeditierung und Epigenetik diskutiert und oft sehr stark kritisiert werden. Daher werden in diesem Kapitel einige dieser Konzepte erklärt. Sowohl für eine ethische Analyse der Epigenetik als auch der Genomeditierung ist das Verständnis und ein kritischer Umgang mit dem grundlegenden Konzept des genetischen Determinismus und weiterer -ismen erforderlich. Eine ausführliche Einführung hierzu findet sich im folgenden Abschnitt (13.2; siehe auch Tab. 2). ${ }^{7}$

5 In der vorliegenden Untersuchung ist die Verwendung des Begriffs „Wissenschaft“, soweit nicht anders angegeben, auf die Naturwissenschaft bezogen.

6 Hierzu zählen Texte, die wissenschaftliche Themen in allgemeinverständlicher Form darstellen, z. B. mediale Wissenschaftstexte oder sogenannte Ratgeber-Literatur, etwa zu Gesundheitsthemen.

7 Zur Epigenetik siehe die Publikation „Epigenetik. Implikationen für die Lebens- und Geisteswissenschaften“ der BBAW, herausgegeben von Jörn Walter und Anja Hümpel (2017). Dort findet sich eine intensive Analyse des Konzepts der Vererbung im Zusammenhang mit der Epigenetik, die wesentlich umfangreicher und differenzierter ist, als es in dem vorliegenden Kapitel geleistet werden kann. Für eine weiterführende Auseinandersetzung mit dieser wichtigen Thematik ist daher auf Walter/Hümpel (2017) zu verweisen. Zu den naturwissenschaftlichen Grundlagen der Epigenetik siehe außerdem Walter/Gasparoni (Kap. 3) sowie Jawaid/Mansuy (Kap. 12). Für eine differenzierte Darstellung der wissenschaftlichen Sichtweisen auf die Rolle von Genen in Geschichte und Gesellschaft siehe Rheinberger/Müller-Wille (Kap. 11). 
Tabelle 2: Analysierte Konzepte

\begin{tabular}{|c|c|c|c|c|c|c|}
\hline & \multirow[t]{2}{*}{ Definition } & \multirow[t]{2}{*}{ Kapitel } & \multicolumn{4}{|c|}{ Zugordnete Diskursbereiche } \\
\hline & & & Wiss. & P.-Wiss. & Ges. & Ethik \\
\hline $\begin{array}{l}\text { genetischer } \\
\text { Essentialismus }\end{array}$ & $\begin{array}{l}\text { Vorstellung, dass der Mensch allein, oder } \\
\text { doch zu einem überwiegenden Teil, durch } \\
\text { seine Gene bestimmt ist }\end{array}$ & 13.2 .1 & & & $x$ & \\
\hline $\begin{array}{l}\text { starker } \\
\text { genetischer } \\
\text { Determinismus }\end{array}$ & $\begin{array}{l}\text { Vorstellung, dass ein Gen fast immer zu der } \\
\text { Ausprägung eines bestimmten Merkmals } \\
\text { (bestimmte körperliche Eigenschaft, Ver- } \\
\text { haltensmerkmal oder Charaktereigenschaft) } \\
\text { führt }\end{array}$ & 13.2 .1 & & & $x$ & \\
\hline $\begin{array}{l}\text { verdeckter Gen- } \\
\text { determinismus }\end{array}$ & $\begin{array}{l}\text { Annahme eines um die Erkenntnisse } \\
\text { der Epigenetik erweiterten genetischen } \\
\text { Determinismus }\end{array}$ & 13.2 .2 & $x$ & $x$ & $x$ & $x$ \\
\hline $\begin{array}{l}\text { epigenetischer } \\
\text { Determinismus }\end{array}$ & $\begin{array}{l}\text { Kombination aus } \\
\text { a) verdecktem Gendeterminismus und } \\
\text { b) der naturwissenschaftlich nicht validierba- } \\
\text { ren Annahme der Möglichkeit einer bewuss- } \\
\text { ten Einflussnahme auf das eigene Epigenom } \\
\text { und das Epigenom zukünftiger Generationen } \\
\text { und daraus abgeleiteter Verantwortung }\end{array}$ & 13.2 .2 & & $x$ & $x$ & $x$ \\
\hline $\begin{array}{l}\text { genetischer } \\
\text { (Daten-) } \\
\text { Exzeptionalismus }\end{array}$ & $\begin{array}{l}\text { ethisch-rechtliche Forderung, dass geneti- } \\
\text { sche Daten einen außergewöhnlich hohen } \\
\text { Schutzanspruch genießen sollten }\end{array}$ & 13.2 .3 & & & $x$ & $x$ \\
\hline $\begin{array}{l}\text { epigenetischer } \\
\text { (Daten-) } \\
\text { Exzeptionalismus }\end{array}$ & $\begin{array}{l}\text { ethisch-rechtliche Forderung, dass epigene- } \\
\text { tische Daten einen außergewöhnlich hohen } \\
\text { Schutzanspruch genießen sollten }\end{array}$ & 13.2 .3 & & & & $x$ \\
\hline
\end{tabular}

Diskursbereiche (Abkürzungen): Wiss. = Naturwissenschaftlicher Diskurs; P.-Wiss. = Populärwissenschaftliche Texte, z. B. Ratgeber; Ges. = Gesellschaftlicher Diskurs; Ethik = Ethischer Diskurs. Das Vorkommen der einzelnen -ismen in den jeweiligen Diskursbereichen ist besonders für den gesellschaftlichen Diskurs hypothetisch. Beobachtungen basieren auf Einschätzungen der Sekundärliteratur zu den jeweiligen Konzepten.

Der darauffolgende Abschnitt (13.3) widmet sich, hierauf aufbauend, der ethischen Analyse von Genomeditierung und Epigenetik. In den Fokus genommen werden dabei Vererbung und Verantwortung, Gerechtigkeit, Sicherheit, die Problematik der Zustimmung/Einwilligung (consent) sowie Auswirkungen auf Embryonen und zukünftige Generationen.

Weitere Aspekte, die sowohl in ethischen Debatten zur Epigenetik als auch zur Genomeditierung auszumachen sind und von uns hier nicht analysiert werden, umfassen u. a.: 
- Angst vor Missbrauch der Erkenntnisse der Epigenetik sowie der Verfahren der Genomeditierung, dies auch hinsichtlich Eugenik und Enhancement, ${ }^{8}$

- Natürlichkeit - wobei wir hierauf zumindest am Rande im Folgenden wiederholt eingehen,

- ein möglicher Zusammenhang von Genom/Epigenom mit dem Konzept der Menschenwürde und die daraus abgeleitete Gefahr der Instrumentalisierung und Autonomieverletzung bei Eingriffen in Genom oder Epigenom.

Da der gegenwärtige Diskurs zu ethischen Fragen der Genomeditierung v. a. Eingriffe in die Keimbahn betrifft, die z. B. am Embryo vorgenommen werden, konzentrieren wir uns für einen Vergleich der Debatten zu Genomeditierung und Epigenetik in Abschnitt 13.3 auf Keimbahneingriffe.

\section{2 -ismen}

Ein wichtiges Anliegen dieses Kapitels ist es, auf die Notwendigkeit einer kritischen Reflexion expliziter, viel öfter aber impliziter -ismen innerhalb des Diskurses sowohl zur Epigenetik als auch zur Genetik und Genomeditierung aufmerksam zu machen. Im Folgenden werden daher die Konzepte genetischer Essentialismus und starker genetischer Determinismus (13.2.1), verdeckter Gendeterminismus und epigenetischer Determinismus (13.2.2) sowie genetischer Exzeptionalismus und epigenetischer Exzeptionalismus (13.2.3) vorgestellt und kritisch analysiert. Jene verschiedenen -ismen sind in unterschiedlichen Diskursbereichen - Wissenschaft, Populärwissenschaft, Gesellschaft, Ethik - unterschiedlich stark ausgeprägt (siehe Tab. 2).

Uns ist bewusst, dass insbesondere der Begriff Determinismus ein sehr starker Begriff ist, der auf eine vollständige Fremdbestimmung schließen lässt (Resnik/Vorhaus, 2006). Wir verwenden diesen und die anderen -ismen lediglich in Anlehnung an im ethischen Diskurs bereits eingeführte Bezeichnungen und möchten nicht etwa selbst proklamieren, dass der Mensch vollständig durch seine Gene oder sein Epigenom bestimmt ist.

Es ist zu beobachten, dass im gesellschaftlichen Diskurs sowohl Vorstellungen eines genetischen als auch eines epigenetischen Determinismus trotz naturwissenschaftlicher Erkenntnisse, die diesen Konzepten entgegenstehen, weiterhin präsent sind. Im Anschluss an eine Einführung in diese verschiedenen -ismen und in Anbetracht der neu-

8 Vgl. den Beitrag von Dieter Birnbacher „Gentechnisches Enhancement“ im „Vierten Gentechnologiebericht" (2018); siehe auch unten (Abschnitt 13.3.1). 
en Möglichkeiten, sowohl in das Genom als zukünftig vielleicht auch in das Epigenom eines Menschen einzugreifen, kann daher gefragt werden:

Können die Verfahren der Genom- und Epigenomeditierung dazu beitragen, die Annahme eines genetischen und epigenetischen Determinismus zu widerlegen, oder befördern diese neuen gentherapeutischen Verfahren im Gegenteil die im gegenwärtigen öffentlichen Diskurs präsenten Vorstellungen von genetischem und epigenetischem Determinismus? Diese Frage ist wichtig für eine kritische Reflexion der hier vorgestellten -ismen.

\subsubsection{Genetischer Essentialismus und starker genetischer Determinismus}

a) Konzept

Der Position eines genetischen Essentialismus liegt die Annahme zugrunde, dass der Genotyp den Phänotyp und das gesamte Wesen eines Menschen, seine Essenz, vollständig determiniert: ,genetischer Essentialismus reduziert das Selbst auf eine molekulare Entität, dabei wird der Mensch, in all seiner sozialen, historischen und moralischen Komplexität, mit seinen Genen gleichgesetzt“ (Nelkin/Lindee, 1995: 2; Übersetzung durch die Autorinnen). Das beinhaltet die Vorstellung, dass der Mensch allein, oder doch zu einem überwiegenden Teil, durch seine Gene bestimmt ist. Dieses Konzept ist aus naturwissenschaftlicher Sicht widerlegt.

Aus naturwissenschaftlicher Perspektive ebenso zweifelhaft wie der genetische Essentialismus und notwendige Voraussetzung desselben ist ein starker genetischer Determinismus. Resnik und Vorhaus (2006) definieren starken genetischen Determinismus als die Annahme, dass ein Gen fast immer zur Ausprägung eines bestimmten Merkmals führt - dies könnte sowohl eine bestimmte körperliche Eigenschaft als auch ein Verhaltensmerkmal oder eine Charaktereigenschaft sein: „Gen $\mathrm{G}$ führt fast immer zu der Entwicklung von Merkmal T. (G erhöht die Wahrscheinlichkeit von T und die Wahrscheinlichkeit von T, gegeben G, ist 95 \% oder größer“ (Resnik/Vorhaus, 2006; Übersetzung durch die Autorinnen). Obgleich mittlerweile von neueren Erkenntnissen der Humangenetik widerlegt, hatte diese „Ein-Gen-ein-Merkmal“-Relation lange Zeit auch innerhalb der Wissenschaft Gültigkeit (Tappeser/Hoffmann, 2004; Schuol, 2016; siehe Rheinberger/ Müller-Wille, Kap. 11). Aufgrund dessen kann davon ausgegangen werden, dass sowohl die Vorstellung eines starken genetischen Determinismus als auch eines genetischen Essentialismus innerhalb der Gesellschaft weiterhin auszumachen ist (Schuol, 2017).

b) Kritik

Die Positionen des genetischen Essentialismus und starken genetischen Determinismus werden innerhalb der Wissenschaftsphilosophie und Wissenschaftsethik scharf kritisiert 
und zurückgewiesen (Dar-Nimrod/Heine, 2011). Dies begründet sich nicht nur daraus, dass sie naturwissenschaftlich widerlegt sind. Die Annahme des genetischen Essentialismus kann vielmehr auch aus ethischer Perspektive problematische Folgen haben. DarNimrod und Heine verweisen darauf, dass die Vorstellung, eine Person sei vollständig durch ihre Gene bestimmt, mitunter dazu führt, Personen mit bestimmten Eigenschaften sowie deren Verwandte stärker zu diskriminieren als dies der Fall wäre, wenn eine nicht-genetische Ursache ihres Verhaltens angenommen würde:

Wissenschaftliche Untersuchungen haben gezeigt, dass stärkere genetische Zuschreibungen für psychische Erkrankungen mit einem verstärkten Wunsch nach sozialer Distanz von Personen mit jenen Erkrankungen [...] und ihren Verwandten verbunden sind. (Dar-Nimrod/Heine, 2011: 808; Übersetzung durch die Autorinnen)

Während hierbei die genetische Verursachung eher negativ aufgefasst wird, ist auch der umgekehrte Fall möglich. Bei der Annahme, dass das Genetische dem Natürlichen, das Natürliche wiederum dem moralisch Guten entspricht (naturalistischer Fehlschluss) und zugleich der Annahme des genetischen Essentialismus, dass der Mensch vollständig durch seine Gene bestimmt ist, wird jedes menschliche Verhalten als moralisch gut angesehen. Raum für Kritik ist nicht gegeben, sondern aufgrund des deterministischen Verständnisses vollständige Legitimation jeder Verhaltensweise anzunehmen. Allenfalls die künstliche Veränderung des menschlichen Genoms würde Raum für eine solche Kritik bieten, da ein genetisch modifiziertes Genom nicht mehr als natürlich und, gemäß eines Natürlichkeitsargumentes, das oben als naturalistischer Fehlschluss bezeichnet wurde, auch nicht mehr als moralisch gut gelten würde, wie es im eingeklammerten Teil des folgenden Zitats heißt:

Ferner gilt, dass man eher geneigt ist, etwas als natürlich zu identifizieren, wenn seine Existenz als von einer zugrundeliegenden genetischen Prädisposition abhängig aufgefasst wird (es sei denn, die Gene sind selbst das Produkt einer künstlichen Veränderung wie im Fall genetisch veränderter Produkte). (Dar-Nimrod/Heine, 2011: 802; Übersetzung durch die Autorinnen)

Würde nach dieser Auffassung auch ein Mensch, der beispielsweise zum Zeitpunkt der Embryonalentwicklung mittels Keimbahneingriff genetisch verändert wurde, als nicht natürlich und mithin (unter Vornahme des naturalistischen Fehlschlusses) als aus moralischer Perspektive schlechter gelten als eine Person, deren Genom nicht in der 
genannten Weise verändert wurde? Gilt Entsprechendes auch für eine Veränderung des Epigenoms? Beides folgt als naturalistischer Fehlschluss möglicherweise aus der Position eines genetischen Essentialismus oder starken genetischen Determinismus und, wie im Folgenden gezeigt wird, aus deren Ergänzung um einen epigenetischen Determinismus. Daher sind sowohl genetischer Essentialismus, starker genetischer Determinismus als auch epigenetischer Determinismus nicht nur aus naturwissenschaftlichen, sondern auch aus ethischen Gründen abzulehnen.

\subsubsection{Verdeckter Gendeterminismus und epigenetischer Determinismus}

a) Konzept

Auch moderatere oder schwächere Formen des genetischen Determinismus, die lediglich davon ausgehen, dass ein Gen manchmal zur Ausprägung bestimmter Merkmale führt (Resnik/Vorhaus, 2006), können, obgleich naturwissenschaftlich korrekt, aus ethischer Sicht problematisch werden, wenn sie um weitere Annahmen ergänzt werden. Dabei handelt es sich um die Annahme, dass durch Wahl der Umweltbedingungen Personen selbst beeinflussen könnten, wann ein Gen zur Ausprägung jener bestimmten Merkmale führt, und diese epigenetische Prägung der Gene dann ggf. auch an zukünftige Generationen weitergegeben werden könne. Unter Umweltbedingungen ist z. B. die Ernährung zu verstehen (Nutri-Epigenetik). Dieses Konzept bezeichnen wir in Anlehnung an Waggoner und Uller (2015) als epigenetischen Determinismus. Auch hierbei werden aus naturwissenschaftlicher Sicht z. T. nicht bestätigte Annahmen zugrunde gelegt. Die nicht bestätigte Annahme einer Weitergabe an zukünftige Generationen basiert u. a. auf Untersuchungen zu Auswirkungen von Mangelernährung auf die nachfolgenden Generationen (Tobi et al., 2014; siehe mit Zusammenfassung weiterer Studien Jawaid/ Mansuy, Kap. 12).

Das Konzept des epigenetischen Determinismus setzt sich somit zusammen aus der Annahme eines um die Erkenntnisse der Epigenetik erweiterten genetischen Determinismus und weiteren Annahmen bezüglich einer bewussten Einflussnahme auf das eigene Epigenom und das Epigenom zukünftiger Generationen und daraus abgeleiteter Verantwortung. Aus naturwissenschaftlicher Sicht problematisch ist insbesondere Letzteres, da eine direkte Einflussnahme auf die eigene Gesundheit und insbesondere die Gesundheit nachfolgender Generationen, vermittelt über das Epigenom, nicht belegbar ist. Zunächst folgen jedoch einige Anmerkungen zu der Annahme eines um die Erkenntnisse der Epigenetik erweiterten genetischen Determinismus. Schuol (2016) bezeichnet diese 
Form des genetischen Determinismus als verdeckten Gendeterminismus, den er wie folgt definiert:

\section{Verdeckter Gendeterminismus:}

„Der Phänotyp wird vom epigenetisch aktivierten Teil des Genoms determiniert.“ (Schuol, 2016: 53)

Dies bedeutet, dass ein Gen nicht unmittelbar zur Ausprägung eines bestimmten Merkmals (phänotypisches Merkmal) führt, wie es der genetische Determinismus behaupten würde, sondern es nur dann zur Ausprägung dieses Merkmals kommt, wenn dieses Gen „epigenetisch aktiviert“ ist. Unter epigenetischer Aktivierung wird dabei eine bestimmte molekulargenetische Konfiguration der DNA verstanden. So ist ein Gen z. B. dann epigenetisch aktiviert, wenn die DNA einen bestimmten Methylierungszustand hat, da eine Transkription von DNA („Ablesen“) nur möglich ist, wenn die Base Cytosin (ein Bestandteil der DNA) nicht methyliert ist, d. h., wenn an das Cytosin keine Methylgruppe gebunden ist (Hanna/Kelsey, 2014). ${ }^{9}$ Gemäß dem gegenwärtigen Epigenetik-Verständnis, das im verdeckten Gendeterminismus zum Ausdruck kommt, bestimmt weiterhin das Genom die phänotypischen Merkmale einer Person.

Sowohl Schuol (2016) als auch Waggoner und Uller (2015) gehen in ihren jeweiligen Analysen des Einflusses der Erkenntnisse der Epigenetik auf das Konzept des genetischen Determinismus in Wissenschaft, Populärwissenschaft und Gesellschaft von einem molekulargenetischen Epigenetik-Begriff, der beispielsweise DNA-Methylierungen umfasst, aus. Beide Analysen kommen zudem zu einem ähnlichen Schluss: Es scheint zunächst, als würde sowohl im populärwissenschaftlichen als auch im wissenschaftlichen Diskurs das Konzept der Epigenetik angeführt, um einen genetischen Determinismus zu widerlegen. Stattdessen besteht dieser hingegen in verdeckter Form fort.

\section{b) Kritik}

Innerhalb der Ethik wird auch das Konzept eines verdeckten Gendeterminismus kritisch gesehen (Schuol, 2017). Zwar ist es naturwissenschaftlich zunächst korrekt, dass, wie Schuol schreibt, der Phänotyp vom epigenetisch aktivierten Teil des Genoms bestimmt wird. D. h., ob ein Gen zur Ausbildung eines bestimmten Merkmals führt, hängt u. a. davon ab, ob, vereinfacht gesagt, das Gen „epigenetisch aktiviert“ ist - was dies aus molekulargenetischer Perspektive bedeutet, wurde oben beschrieben. Problematisch ist

9 Hier setzt die Epigenomeditierung an, die das Ziel hat, die Transkription bestimmter Gene zu beeinflussen, indem sie das Epigenom, z. B. den Methylierungszustand der DNA, ändert (siehe Tab. 1). 
der verdeckte Gendeterminismus aber einerseits dann, wenn man davon ausgeht, dass der Mensch nur durch sein „epigenetisch aktiviertes“ Genom und nicht etwa auch durch seine Sozialisation beeinflusst wird. Andererseits ist der verdeckte Gendeterminismus auch ethisch problematisch, nämlich dann, wenn er zum epigenetischen Determinismus wird. Wir definieren den epigenetischen Determinismus folgendermaßen:

Epigenetischer Determinismus wird definiert als eine Kombination aus:

a) verdecktem Gendeterminismus und

b) der naturwissenschaftlich nicht validierbaren Annahmen der Möglichkeit einer bewussten Einflussnahme auf das eigene Epigenom und das Epigenom zukünftiger Generationen und daraus abgeleiteter Verantwortung.

Annahme b) ist in populärwissenschaftlichen Texten, wie z. B. Ratgebern oder medialen Wissenschaftstexten, zu finden (Schuol, 2017) und nimmt darüber Einfluss auf gesellschaftliche Vorstellungen zur Epigenetik. Diese Annahme einer Verantwortung für die eigene Gesundheit und die Gesundheit zukünftiger Generationen aufgrund der Möglichkeit, durch eine bewusste Wahl von Umweltbedingungen das Epigenom zu verändern und somit Einfluss auf das „Ablesen“ bestimmter Gene zu nehmen, wobei diese Veränderungen des Epigenoms über mehrere Generationen hinweg stabil sein könne, kann aus naturwissenschaftlicher Sicht nicht bestätigt werden. Es sprechen im Gegenteil sogar einige Gründe gegen sie. Z. B. ist eine Art epigenetischer Vererbung beim Menschen dadurch ausgeschlossen, dass das Epigenom während der Entwicklung von Ei-/Samenzelle und Embryo zweimal fast vollständig neu konfiguriert wird (sogenannte epigenetische Reprogrammierung). Die naturwissenschaftlich nicht bestätigte Annahme b) wird in Diskursen zur Epigenetik dennoch mitunter vertreten.

Das Konzept des epigenetischen Determinismus ist daher eine Beschreibung der gegenwärtigen Diskussionen zu Genetik und Epigenetik. Da es auf naturwissenschaftlich nicht belegten Voraussetzungen beruht, sind die Schlussfolgerungen hinsichtlich der Verantwortung für die eigene Gesundheit und die Gesundheit der eigenen Kinder bzw. weiterer nachfolgender Generationen nicht berechtigt und aus ethischer Sicht problematisch. Ein epigenetischer Determinismus kann mitunter auch in ethischen Texten identifiziert werden (vgl. unten, Abschnitt 13.3.2). Hierbei sind Argumente, die sich auf Grundlage der Epigenetik für eine Verantwortung für die Gesundheit zukünftiger Generationen aussprechen, dann besonders kritisch zu sehen, wenn sie von einer naturwissenschaftlich zumindest strittigen Annahme einer bewussten, direkten epigenetischen Einflussnahme, z. B. durch bestimmtes Ernährungsverhalten, auf die eigenen Nachkommen ausgehen. Denn wesentliche Voraussetzung für eine fundierte ethische 
Debatte ist aber, dass sie sich am aktuellen Stand der Wissenschaft orientiert. Der aktuelle Stand der Wissenschaft liefert keinen eindeutigen Beleg für die intergenerationale Vererbbarkeit erworbener epigenetischer Veränderungen beim Menschen. ${ }^{10}$ Daher ist es erstaunlich, wie lange sich Argumente zur Verantwortung für nächste Generationen in der ethischen Diskussion der Epigenetik halten und wiederholt werden.

Eine Sensibilisierung für die Konzepte verdeckter Gendeterminismus und epigenetischer Determinismus hat zum Ziel, jene aus ethischer Sicht problematischen Konsequenzen zu revidieren. Schuol (2017) verweist auf diese Problematik von Verantwortungszuschreibungen in populärwissenschaftlichen Diskursen zur Epigenetik. Wir werden in Abschnitt 13.3 ausführen, dass gerade aufgrund der deterministischen Vorstellung zur Epigenetik ethische Diskurse zu Epigenetik solchen zur Genomeditierung inhaltlich nahestehen.

13.2.3 Genetischer Exzeptionalismus und epigenetischer Exzeptionalismus mit Blick auf die informationelle Selbstbestimmung

a) Konzept

Obgleich wir sowohl den starken genetischen Determinismus als auch den epigenetischen Determinismus als aus naturwissenschaftlicher und ethischer Sicht problematisch bezeichnet haben, ist die Idee, dass genetische und vielleicht auch epigenetische Daten von Personen oder Bevölkerungsgruppen einen besonderen rechtlichen Schutz genießen sollten, nicht vollkommen unbegründet. ${ }^{11}$ Diese Idee wird auch als genetischer oder epigenetischer Exzeptionalismus bezeichnet, wobei der genetische Exzeptionalismus das ältere und verbreitetere dieser beiden Konzepte bzw. ethisch-rechtlichen Forderungen ist. Er bezieht sich auf die Forderung eines besonderen Schutzanspruchs genetischer Daten (z. B. Ganz-Genom-Sequenzierungsdaten, die im Rahmen von Forschung oder genetischer Diagnostik gewonnen werden). Der genetische Exzeptionalismus findet sich innerhalb ethischer Debatten, wird z. T. aber auch kritisiert. Wir untersuchen im Folgenden, ob eine Kritik am genetischen Exzeptionalismus berechtigt ist. Zudem erwägen wir, einen genetischen Exzeptionalismus um das Erfordernis, auch epigenetische Daten besonders zu schützen, zu ergänzen und somit den genetischen um einen epigenetischen Exzeptionalismus zu erweitern.

10 Zur Gegenposition und Darstellung des gegenwärtigen Forschungsstandes zu epigenetischer Vererbung siehe Jawaid/Mansuy (Kap. 12).

11 Zu ethischen Fragen in Bezug auf medizinische Daten siehe auch Molnár-Gábor/Merk zu Hirndaten (Kap. 16) sowie Winkler/Prainsack zu Big Data und personalisierter Medizin (Kap. 17). 
b) Kritik

b.i. Was spricht gegen einen genetischen und epigenetischen Exzeptionalismus mit Blick auf genetische Daten?

Mit der Kritik am genetischen Essentialismus ist auch die medizinethische Kritik am Konzept des genetischen Exzeptionalismus innerhalb von Debatten um die Sonderstellung genetischer gegenüber nicht-genetischer medizinischer Information verbunden, insbesondere in Bezug auf einen höherrangigen rechtlichen Schutzanspruch genetischer im Vergleich zu nicht-genetischen Daten.

Denn die Annahme eines genetischen Exzeptionalismus ist plausibel, wenn man Folgendes voraussetzt: Personen mit bestimmten genetischen Merkmalen könnten von Dritten diskriminiert werden, wenn diese Dritten das Konzept des genetischen Essentialismus vertreten und Personen mit bestimmten genetischen Merkmalen deshalb diskriminieren. Dritte nehmen also an, dass die invariablen genetischen Merkmale das Wesen dieser Personen (nahezu) vollständig bestimmten (genetischer Essentialismus). Um eine auf dieser Annahme basierende Diskriminierung zu vermeiden, müssten genetische Daten im Vergleich zu anderen medizinischen oder persönlichen Daten vor dem Zugriff Dritter besonders geschützt werden, so die These des genetischen Exzeptionalismus (Green/ Botkin, 2003). Da sich jedoch der genetische Essentialismus als falsch herausgestellt hat, wäre auch die Annahme, dass genetische Daten einen höheren Schutzanspruch genießen sollten als nicht-genetische Daten, naturwissenschaftlich unplausibel.

Denn nicht nur genetische, sondern z. B. auch epigenetische und weitere Informationen über Personen und Personengruppen haben eine hohe Aussagekraft, z. B. in Bezug auf sensible Merkmale dieser Personen. Dupras und Bunnik (2021) nennen hierfür viele Beispiele, die u. a. die Reidentifizierbarkeit von Personen anhand ihrer genetischen und weiterer, nicht-genetischer Daten betreffen. Sie sprechen sich explizit gegen einen genetischen Exzeptionalismus aus und plädieren stattdessen für einen „multi-omic contextualism“. Bei diesem Kontextualismus-Ansatz hängt der Schutz verschiedener Forschungsdaten nicht davon ab, welchem Datentyp sie zuzuordnen sind. Es geht stattdessen darum, wie sensibel die Daten jeweils sind und wie schlimm die Folgen eines Datenmissbrauchs wären.

In früheren Versionen ihres Kontextualismus-Modells haben Dupras und Kollegen bereits darauf verwiesen, dass der besondere Schutzanspruch genetischer Daten (genetischer Exzeptionalismus) durch einen besonderen Schutzanspruch epigenetischer Daten ergänzt werden muss, da diese ebenso sensibel sind (Dupras et al., 2018; Dupras et al., 2020). Auch Dupras' und Bunniks neueres Kontextualismus-Modell basiert v. a. auf einer Analyse genetischer und epigenetischer Daten, dennoch weisen Dupras und Bunnik (2021) implizit nicht nur den genetischen, sondern auch den epigenetischen Exzep- 
tionalismus zurück. Gegen diese beiden Konzepte spricht, dass auch andere Datentypen, abhängig vom Kontext, eine ähnlich hohe Sensibilität und deshalb einen ähnlich hohen Schutzanspruch haben wie genetische und epigenetische Daten.

\section{b.ii. Was spricht für einen genetischen und epigenetischen Exzeptionalismus?}

Für die Forderung eines genetischen Exzeptionalismus spricht zunächst die - auch ohne, dass ein starker genetischer Determinismus vertreten werden muss - völlig berechtigte Annahme, dass genetische Daten eine besonders hohe Aussagekraft haben. Denn einerseits lassen sie oft prädiktive Aussagen zu, die z. B. das Risiko für das Auftreten einer bestimmten Erkrankung betreffen. Andererseits haben sie eine Aussagekraft, die sich über den Bereich des Individuums auch auf genetisch verwandte Familienmitglieder hinaus erstreckt. Dupras und Bunnik (2021) bemerken allerdings, dass diese beiden Eigenschaften (Prädiktivität und Aussagekraft in Bezug auf Dritte) auch auf nicht-genetische Daten zutreffen könnten. Als Beispiel nennen sie epigenetische Varianten, die verschiedene Mitglieder einer sozialen Gemeinschaft teilen (Dupras/Bunnik, 2021: 5). Zudem könne es sein, dass eine Kombination aus epigenetischen und genetischen Daten das Risiko einer Reidentifizierbarkeit erhöht, weshalb zu erwägen ist, einen genetischen um einen epigenetischen Exzeptionalismus zu ergänzen.

b.iii. Implikationen von starkem genetischem und epigenetischem Determinismus für genetischen und epigenetischen Exzeptionalismus

Garrison und Kollegen (2019: 53) weisen darauf hin, dass der genetische Exzeptionalismus gegenwärtig innerhalb des ethischen Diskurses nur noch wenige Befürworter findet. Für die Forderung eines genetischen Exzeptionalismus spricht allerdings das folgende Argument.

Es ist zwar richtig, dass der genetische Determinismus naturwissenschaftlich widerlegt ist, aber die Ethik sollte sich von dem Thema nicht zu früh abwenden. Denn wenn er im populärwissenschaftlichen Diskurs kolportiert wird und weiterhin viele Menschen glauben, dass unsere Gene uns weitgehend bestimmen, dann verhalten sie sich auch so. Das bedeutet, dass aufgrund dieser Fehlwahrnehmung weiterhin Menschen diskriminiert werden könnten. Wenn dem so ist, muss sich die Ethik mit den Auswirkungen dieser falschen Annahmen weiterhin beschäftigen.

Um für einen besonderen Schutzanspruch genetischer Versorgungs- und Forschungsdaten zu argumentieren, ist es also lediglich erforderlich, anzunehmen, dass die Vorstellung eines starken genetischen Determinismus oder genetischen Essentialismus in der Gesellschaft vorherrsche. Genauer, dass diese bei Dritten vorherrsche, welche Personen auf Grundlage derer genetischer Merkmale diskriminieren könnten. Es ist 
hingegen keine notwendige (jedoch $u$. U. hinreichende) Voraussetzung, selbst Vertreter der Annahme des starken genetischen Determinismus oder Essentialismus zu sein, um den genetischen Exzeptionalismus zu befürworten. Gleiches gilt überdies für ethisch problematische Vorstellungen zur Epigenetik. Solange die Meinung vorherrscht, dass aufgrund epigenetischer Steuerbarkeit der Genregulation Personen für ihre Gesundheit selbst verantwortlich zu machen sind, muss die Ethik sich mit den gesellschaftlichen Folgen dieser Annahme auseinandersetzen. Dies würde die Forderung beinhalten, nicht nur genetische, sondern auch epigenetische Daten unter einen besonderen Schutz zu stellen, um eine Diskriminierung aufgrund dieser Daten zu vermeiden (epigenetischer Exzeptionalismus).

Wichtig ist dabei auch der Aspekt der Vererbung. Während ein genetischer Determinismus zumindest insofern berechtigt erscheint, als genetische Information tatsächlich vererbt wird, scheint die Annahme der Vererbbarkeit als Teil des epigenetischen Determinismus zwar auch im ethischen Diskurs zur Epigenetik eine entscheidende Funktion zu haben, unterscheidet aber doch die Bedeutung von genetischer und epigenetischer Information und unterstreicht möglicherweise den exzeptionellen Schutzanspruch von genetischen Daten (genetischer Exzeptionalismus).

Die Forderung eines genetischen und epigenetischen Exzeptionalismus müsste von dem Versuch begleitet sein, die Öffentlichkeit zu einem kritischen Umgang mit den wissenschaftlich und ethisch problematischen Annahmen eines starken genetischen und epigenetischen Determinismus zu sensibilisieren. Denn falsche Annahmen in Bezug auf Epigenetik führen zu ethisch problematischen Zuschreibungen von Verantwortung (epigenetischer Determinismus). Falsche Annahmen in Bezug auf die Genetik (starker genetischer Determinismus) können z. B. zu einem naturalistischen Fehlschluss führen, der alles Genetische als natürlich und alles Natürliche als gut bezeichnet oder sie können in der Diskriminierung von Personen mit bestimmten genetischen Eigenschaften resultieren. Ohne dieses kritische Verständnis der hier untersuchten Konzepte ist es immerhin vorstellbar, dass die Forderung von genetischem und epigenetischem Exzeptionalismus in Bezug auf den Umgang mit genetischen und epigenetischen Daten die problematischen Annahmen von starkem genetischem und epigenetischem Determinismus noch verstärken könnte. Daher möchte das vorliegende Kapitel für einen kritischen Umgang mit expliziten und impliziten -ismen in Bezug auf Vorstellungen zu Genetik, Epigenetik und Gentechnik sensibilisieren. 


\subsection{Ethischer Diskurs zu Epigenetik und Genomeditierung - Überschneidungen und Differenzen anhand zentraler Aspekte}

\subsubsection{Determinismus}

Das deterministische Verständnis in Diskursen zu Genetik und Epigenetik hat zur Folge, dass Debatten zu ethischen Aspekten der Epigenetik inhaltlich starke Parallelen zum ethischen Diskurs über den therapeutischen Einsatz der Genomeditierung am Menschen aufweisen. Die Parallele liegt dabei v. a. in der Relevanz der Bezugnahme auf eine mögliche genetische oder (naturwissenschaftlich nicht bestätigte) epigenetische Vererbung und daraus abgeleiteter Verantwortung für zukünftige Generationen.

In der ethischen Diskussion zur Genomeditierung begegnen wir divergierenden Positionen hinsichtlich der Fragestellung, wie der Aspekt der Vererbbarkeit von Keimbahneingriffen ethisch beurteilt werden sollte (siehe auch Fehse et al., Kap. 9). Dabei stehen Positionen, die von einem moralischen Imperativ zur Erforschung therapeutischer oder sogar verbessernder ${ }^{12}$ Anwendungen der Keimbahn-Genomeditierung ausgehen (Savulescu et al., 2015), solchen Positionen gegenüber, die ein Moratorium hinsichtlich der Erforschung der Genomeditierung an Embryonen fordern. ${ }^{13}$ Mit einer therapeutischen Veränderung des Genoms sind somit große Hoffnungen auf positive Auswirkungen verbunden, die über mehrere Generationen hinweg anhalten (z. B. die Hoffnung, eine innerhalb einer Familie in der Vergangenheit gehäuft aufgetretene genetisch bedingte Erkrankung nicht mehr weiterzuvererben). Doch auch die Angst vor schwerwiegenden negativen Konsequenzen, die ebenfalls an mehrere Generationen weitergegeben werden könnten, ist in Debatten zur Genomeditierung zentral. Diese extremen Positionen (Imperativ vs. Moratorium) innerhalb des Diskurses zur Genomeditierung von Embryonen erklären sich z. T. aus der besonderen Bedeutung, die einem Eingriff in das Genom wegen der Annahme eines genetischen Determinismus zukommt.

\subsubsection{Komplexität der Fragestellung und Auflösung von Grenzen}

Diskursen zu Genetik und Genomeditierung sowie zu Epigenetik gemeinsam ist ferner die Schwierigkeit und Vielschichtigkeit der Aufgabe, diese Bereiche ethisch zu bewer-

12 Vgl. zum Konzept des verbessernden Keimbahneingriffs den Beitrag von Dieter Birnbacher „Gentechnisches Enhancement“ im „Vierten Gentechnologiebericht“ (2018).

13 Von den 61 von Brokowski (2018) untersuchten Stellungnahmen zur Genomeditierung, die zwischen 2015 und 2018 erschienen sind, sprechen sich nur 5 \% explizit gegen ein solches Moratorium aus. 
ten. Dies ergibt sich aus der Komplexität der naturwissenschaftlichen Voraussetzungen, v. a. in Bezug auf die Auflösung klarer Kausalitätsbeziehungen zwischen Gen bzw. Genom, Epigenom und Umwelt. Denn es besteht Unklarheit darüber, was in Folge jener Grenzauflösungen unter den Begriff „Vererbung“ fällt. So analysieren beispielsweise Lewens (2020) und Guttinger (2020) Genomeditierung aus ethischer Perspektive mit explizitem Bezug auf Erkenntnisse der Epigenetik und begründen die Komplexität der Fragestellung einer ethischen Analyse der Genomeditierung mit einem post-genomischen Verständnis von Vererbung.

Auch ohne Hinweis auf einen Zusammenhang von genetischer und epigenetischer Verursachung könnte bereits die Möglichkeit, das Genom oder auch das Epigenom mittels Gentechnik gezielt zu verändern, dazu beitragen, Konzepte von genetischer Vererbung und epigenetischer Verursachung zu verkomplizieren und vormals bestehende Grenzen aufzulösen. Dies ergibt sich zumindest dann, wenn der Eingriff in Genom oder Epigenom als künstliche Veränderung natürlicher genetischer und epigenetischer Funktionszusammenhänge bezeichnet wird. Welche ethische Relevanz die Differenz von Natürlichkeit und Künstlichkeit hierbei hat, hängt u. a. davon ab, ob ein naturalistischer Fehlschluss vorgenommen wird (Abschnitt 13.2.1). Zudem wurde innerhalb der Medizinethik bereits die Frage diskutiert, ob eine Veränderung der genetischen Information im Zusammenhang mit Verfahren der assistierten Reproduktion einen Einfluss auf das Konzept der genetischen Elternschaft hat (Piotrowska, 2019). Da auch die Genomeditierung als Verfahren der assistierten Reproduktion in Zukunft möglicherweise in Betracht kommt (National Academies of Sciences et al., 2020) und hierbei ebenfalls genetische Information verändert wird, könnte Piotrowskas Fragestellung in dem Artikel „Why is an egg donor a genetic parent, but not a mitochondrial donor?" (Warum gilt eine Eizell-Spenderin als genetisches Elternteil, aber nicht eine Mitochondrien-Spenderin?) ${ }^{14} \mathrm{um}$ die Frage ergänzt werden, ob auch Genom- und Epigenomeditierung an Embryonen oder Feten Konsequenzen für das Konzept genetischer Elternschaft haben.

Aus der Auflösung der Grenzen von genetischen und nicht-genetischen Kausalitätsbeziehungen folgt eine nicht nur aus naturwissenschaftlicher, sondern auch aus ethischer Sicht komplexe Ausgangssituation für die Analyse von Epigenetik sowie Genomeditierung. Laut Falomir (2018) ergibt sich für den Epigenetik-Diskurs daraus die Notwendigkeit, für eine wissenschaftliche Analyse der Epigenetik auch die Grenzen der

14 Mitochondrien sind Zellorganelle, die für die Energieversorgung der Zelle verantwortlich sind. Sie werden über die Eizelle der Mutter vererbt und enthalten ihre eigene DNA (mtDNA). Bei bestimmten Erkrankungen, die durch funktionsunfähige Mitochondrien verursacht werden, kann bei einer künstlichen Befruchtung auf verschiedene Weise dafür gesorgt werden, dass die Mitochondrien gegen solche einer Spenderin ausgetauscht werden. 
Disziplinen aufzulösen. Gleiches gilt für Diskussionen zur Genomeditierung, die insbesondere aufgrund der Vorstellung eines (verdeckten) genetischen Determinismus immer zugleich aus mehreren disziplinären Blickwinkeln zu betrachten sind, damit die deterministischen Vorstellungen sowohl naturwissenschaftlich geprüft als auch ihre ethischen Konsequenzen aufgezeigt werden können. Das Erfordernis einer interdisziplinären Herangehensweise ist somit Diskursen zu Epigenetik wie zu Genom-Editierung gemein.

\subsubsection{Epigenetik-Diskurs - Vererbung, Verantwortung, Gerechtigkeit}

a) Vererbung und Verantwortung

Der ethische Diskurs zur Epigenetik setzt sich mit der Frage nach Verantwortung auseinander. So ist in einem Forschungsbericht der BBAW zur Epigenetik, auf den eingangs verwiesen wurde, zu lesen: „Besondere Brisanz hat die Frage, in welchem Maß es eine epigenetische Verantwortung des Individuums für die Gestaltung der Lebensumstände nachfolgender Generationen gibt“" (Walter/Hümpel, 2017: 28). Schuol verweist in seiner ethischen Analyse des populärwissenschaftlichen Diskurses zur Epigenetik ebenfalls auf die Zentralität der Frage nach Verantwortung:

Auf Seiten populärwissenschaftlicher Ratgeber werden [...] drei Hauptthemen diskutiert. [...] 1. der durch die Epigenetik eingeleitete Epochenwandel und die Ablösung von einem Genfatalismus, 2. das dadurch beförderte Thema lebensstilbedingter Steuerbarkeit der Generegulation und 3. sich in Folge dieser Steuerbarkeit ergebende Verantwortungsbereiche. [...] Die Aussage, dass mit der Epigenetik eine transgenerationale Verantwortung verbunden sei, wurde eingegrenzt: Gegen eine epigenetische Vererbung beim Menschen sprechen mehrere Gründe [...]. (Schuol, 2017: 368-371; Hervh. i. O.)

Was Schuol hier beschreibt, entspricht dem oben beschriebenen Konzept des epigenetischen Determinismus. Sowohl Schuol als an anderer Stelle auch Walter und Hümpel verweisen auf die Probleme der Annahme eines epigenetischen Determinismus, da dieser auf naturwissenschaftlichen Voraussetzungen beruht, die nicht bestätigt werden können. Dennoch findet sich die Annahme einer ,lebensstilbedingte[n] Steuerbarkeit der Genregulation“, worunter, wie oben dargelegt, beispielsweise die Ernährung fällt, gekoppelt mit der Annahme einer „epigenetische[n] Vererbung“, nicht nur im populärwissenschaftlichen Bereich und in Folge dessen auch innerhalb des gesellschaftlichen 
Diskurses. Sondern ein solcher epigenetischer Determinismus ist auch innerhalb des ethischen Diskurses zur Epigenetik auszumachen.

Ethische Analysen zur Epigenetik kommen somit z. T. zu sehr unterschiedlichen Ergebnissen. Entweder gehen sie von epigenetischer Vererbung und Verantwortung für zukünftige Generationen grundsätzlich aus (z. B. Bode, 2016). Oder sie weisen generationenübergreifende Verantwortung explizit zurück (z. B. Schuol, 2016; Huang/ King, 2018). Nur Letzteres entspricht, wie oben gezeigt, dem aktuellen Stand der Wissenschaft (für eine andere Darstellung siehe Jawaid/Mansuy, Kap. 12). Die Annahme epigenetischer Vererbung und Verantwortung für zukünftige Generationen ist somit hinfällig, solange sie naturwissenschaftlich nicht belegt ist.

\section{b) Gerechtigkeit}

Im Epigenetik-Diskurs wird der sich aus der Annahme des epigenetischen Determinismus ergebende Aspekt der Verantwortung für zukünftige Generationen (siehe vorheriger Abschnitt) auch als intergenerationale Gerechtigkeit bezeichnet. Da der epigenetische Determinismus zudem davon ausgeht, dass über eine bewusste Wahl der Umweltbedingungen ein Einfluss auf das Epigenom und somit auf die eigene Gesundheit möglich ist, wird im Epigenetik-Diskurs auch die Forderung nach Umwelt-Gerechtigkeit formuliert (gesunde Umweltbedingungen für alle). Ergänzt um die Forderung nach einem gerechten Zugang zu Gesundheitsversorgung ergibt sich die Trias: „Umweltgerechtigkeit, intergenerationale Gerechtigkeit und gerechter Zugang zu Gesundheitsversorgung “. ${ }^{15}$

Der Aspekt der Umweltgerechtigkeit bezeichnet einen zentralen Unterschied zwischen den Diskursen zu Genomeditierung und zu Epigenetik. Denn hier wird deutlich, dass Diskussionen zur Epigenetik sich eher im Bereich der Public-Health-Ethik verorten lassen, während die Debatte über die Genomeditierung zwar mit dem Verweis auf distributive Gerechtigkeit z. T. auch in diesem Bereich geführt wird, jedoch überwiegend eine individualethische Perspektive einnimmt. So ist die dreiteilige Forderung nach Umwelt-Gerechtigkeit, intergenerationaler Gerechtigkeit und gerechtem Zugang zu Gesundheitsleistungen v. a. als Forderung auf institutioneller Ebene zu verstehen. Verantwortung für die eigene Gesundheit und die Gesundheit zukünftiger Generationen wird daher bei Dupras und Kollegen nicht dem Einzelnen, sondern staatlichen Institutionen zugeschrieben. Denn es wäre falsch, die Armen der Gesellschaft dafür verantwortlich zu machen, dass sie unter ungesunden Umweltbedingungen leben, „Faktoren, die, über die Epigenetik, ihre eigene Gesundheit und die ihrer Kinder negativ

15 „environmental justice, intergenerational equity and equitable access to healthcare“ (Dupras/ Ravitsky, 2016: 538; Übersetzung durch die Autorinnen). 
beeinflussen können“ (Dupras et al., 2014: 333; Übersetzung durch die Autorinnen). Hier ist erneut darauf zu verweisen, dass die Annahme einer Art epigenetischer Vererbung beim Menschen, die in diesem Zitat zum Ausdruck kommt, aus naturwissenschaftlicher Sicht nicht belegt werden kann.

\subsubsection{Genomeditierungs-Diskurs - Sicherheit, Einwilligung, zukünftige Generationen}

Auch in Debatten zur Genomeditierung ist die Forderung nach einem gerechten $\mathrm{Zu}$ gang zu vielversprechenden Verfahren der Gentherapie zentral (van Dijke et al., 2018). Darüber hinaus ergibt sich v. a. mit der Relevanz, die dem Aspekt der Vererbung zukommt, eine weitere Gemeinsamkeit zu Diskursen zur Epigenetik. Debatten der Genomeditierung befassen sich überwiegend mit ethischen Fragen zu Keimbahneingriffen und fokussieren seit ihren Anfängen auf die Verantwortung für zukünftige Generationen (Agius/Busuttil, 1998). So begründet die zuvor genannte Forderung eines Moratoriums der Erforschung von Keimbahn-Genomeditierung sich aus der Problematik, dass die Folgen eines Eingriffs in das Genom auf Ebene der Keimbahn (z. B. am Embryo) an zukünftige Generationen vererbt werden können.

\section{a) Sicherheit}

Da die Auswirkungen einer Genomeditierung mit therapeutischer Intention vorab nicht vollständig bekannt sind, stellt die Weitergabe negativer Effekte an unzählige Nachfolgegenerationen eine gravierende Sicherheitsproblematik der Keimbahn-Genomeditierung dar. Aus diesem Grund wird regelmäßig als Voraussetzung eines solchen Eingriffs hinreichende Sicherheit genannt (Holm, 2019). Ein Beispiel hierzu findet sich in der folgenden Empfehlung aus einer Stellungnahme US-amerikanischer und britischer Wissenschaftsgesellschaften zu Keimbahneingriffen mittels Genomeditierung:

Vor jeglichem Versuch, eine Schwangerschaft mit einem Embryo, der durch Genomeditierung verändert wurde, herbeizuführen, muss durch präklinische Forschung belegt werden, dass vererbbare Genomeditierung am Menschen (heritable human genome editing, HGGE) mit hinreichend hoher Effizienz und Präzision durchgeführt werden kann, um einen klinischen Nutzen zu generieren. Für jeden ersten Einsatz der HGGE sollte die präklinische Evidenz zur Sicherheit und Wirksamkeit auf der Untersuchung einer signifikanten Kohorte editierter menschlicher Embryonen beruhen und sollte demonstrieren, dass der Prozess in der Lage ist, mit hoher Exaktheit eine geeignete Anzahl von Embryonen zu generieren und auszuwählen, die: 
- beabsichtigte Editierung(en) und keine anderen Veränderungen an der/den genetischen Zielsequenz(en) aufweisen;

- keine zusätzlichen Varianten an Off-Target-Stellen haben, die durch den EditierungsProzess entstanden sind - das heißt, die Gesamtzahl neuer genomischer Varianten sollte nicht signifikant von der Zahl bei vergleichbaren nicht-editierten Embryonen abweichen;

- keine Evidenz von Mosaizismus, entstanden durch den Editierungs-Prozess, aufweisen;

- von geeignetem klinischem Grad sind, um eine Schwangerschaft herbeizuführen; und

- keine Aneuploidie-Raten haben, die höher sind, als es, basierend auf herkömmlichen Verfahren der assistierten Reproduktion, erwartet werden kann.

(National Academy of Sciences et al., 2020: Empfehlung 5; Übersetzung durch die Autorinnen)

b) Informierte Einwilligung

Im ethischen Diskurs zur Genomeditierung besonders zentral ist neben der Sicherheit der Aspekt, dass ein Keimbahneingriff einerseits notwendigerweise ohne Zustimmung des Betroffenen durchgeführt wird, insofern er an Embryonen erfolgt. Denn Embryonen sind noch nicht zustimmungsfähig. Andererseits hat der Eingriff aufgrund der Vererbbarkeit auch Auswirkungen auf potenzielle Nachkommen dieser Embryonen. Diese können dem Eingriff ebenfalls nicht zustimmen, da sie noch nicht existieren. In englischsprachigen Texten zu ethischen Fragen von Keimbahneingriffen wird dieser Aspekt unter den Begriff des consent gefasst, der gewöhnlich mit informierter Einwilligung übersetzt wird: ,issues of consent and threats to the autonomy of future generations are coming to the forefront of the debate" (Fragen der informierten Einwilligung und der Bedrohung der Autonomie zukünftiger Generationen rücken in den Vordergrund der Debatte) (Mintz et al., 2019; Übersetzung durch die Autorinnen).

In Zusammenhang mit dem Problem der Sicherheit erscheint die consent-Problematik für die Genomeditierung an Embryonen besonders relevant. Denn es ist offenbar unmöglich, das Problem der Unsicherheit über möglicherweise gravierende negative Folgen zu lösen, bevor die Technik erstmals an menschlichen Embryonen eingesetzt wird (Guttinger, 2020).

Das Problem, dass ein medizinischer Eingriff manchmal ohne Zustimmung des Betroffenen durchgeführt werden muss, ist zwar aus anderen Bereichen bekannt, z. B. bei der Behandlung von Kindern, bewusstlosen Personen und anderen Nicht-Einwilligungsfähigen. In der Debatte zur Genomeditierung ist die Zustimmungsproblematik aber besonders zentral, da es sich bei Embryonen und zukünftigen Generationen nicht 
um typische Fälle handelt, in denen consent nicht eingeholt werden kann. Denn für Nicht-Einwilligungsfähige gilt i. d. R.:

a. zum Zeitpunkt eines Eingriffs, in den sie nicht einwilligen können, existieren sie bereits;

b. sie haben Stellvertreter, die in ihrem Interesse eine Entscheidung treffen können (bei Kindern sind dies häufig deren Eltern);

c. der Eingriff, in den sie nicht einwilligen können, ist mit besser prognostizierbaren Risiken verbunden als es ein erster klinischer Einsatz der Genomeditierung wäre (Aspekt der Sicherheit, siehe oben);

d. ihre Existenz hängt nicht von dem Eingriff selbst ab.

Diese vier Voraussetzungen treffen sowohl auf Embryonen, an denen eine Genomeditierung vorgenommen wird, als auch auf deren potenzielle Nachkommen, d. h. auf zukünftige Generationen, i. d. R. nicht zu. Ein vererbbarer Eingriff in das Genom von Embryonen oder Keimzellen ist somit selbst dann problematisch, wenn hinreichende Sicherheit vorausgesetzt werden könnte, da dann noch immer drei der genannten, auf consent bezogenen Aspekte ( $a, b$ und $d$ ) nicht gelten.

\section{c) Auswirkungen auf Embryonen und zukünftige Generationen}

Trotz der Zentralität des consent-Problems im gegenwärtigen Diskurs zur Genomeditierung gibt es auch Kritik an diesem Aspekt. Denn diese Position unterlasse es, darauf zu verweisen, wie ein solcher consent eingeholt werden könne (Cavaliere, 2018: 218). Innerhalb von Debatten zur Genomeditierung wird jedoch das Problem der Vererbbarkeit von Keimbahneingriffen auch aus anderen Gründen als der Nicht-Zustimmungsfähigkeit bzw. Nicht-Einwilligungsfähigkeit von Embryonen und zukünftigen Generationen als problematisch angesehen.

Abhängig vom moralischen Status des Embryos könnte es unzulässig sein, den Embryo zur Erforschung der Genomeditierung zu verwenden und im Anschluss zu verwerfen. Doch auch, wenn der Embryo nach der Genomeditierung nicht verworfen, sondern zur Herbeiführung einer Schwangerschaft einer Frau implantiert wird, ist ein Eingriff in dessen Genom aus ethischen Gründen möglicherweise problematisch. Z. T. begründet sich dies aus dem Recht des Embryos und dessen potentieller Nachkommen auf eine offene Zukunft. ${ }^{16}$ Damit verbunden ist die Problematik eines negativen Ein-

16 Zurückgehend auf Joel Feinberg's Konzept eines „right of the child of an open future“, vgl. im Genomeditierungs-Diskurs z. B. Mintz et al. (2019). 
flusses auf das Verhältnis zwischen den Generationen, indem die eine Generation auf die Zusammensetzung des Genoms der anderen Generation Einfluss nehme, was aus ethischer Sicht negativ zu beurteilen sei. ${ }^{17}$

Der moralische Status des Embryos ist nicht nur für Debatten zur Genomeditierung auf Keimbahnebene relevant, sondern auch in Bezug auf die sogenannte pränatale Diagnostik (PND) und Präimplantationsdiagnostik (PID) von Bedeutung. Bei der PID werden Embryonen genetisch untersucht und daraufhin diejenigen unter ihnen ausgewählt und mit dem Ziel einer Schwangerschaft implantiert, die vermeintlich „gesund“ sind. Im ethischen Diskurs zur Genom-Editierung wird mitunter die Annahme vertreten, dass die Genomeditierung eine Alternative zur PID darstellen könnte. Einige Arbeiten untersuchen daher PID und Genomeditierung vergleichend und kommen zu unterschiedlichen Ergebnissen hinsichtlich der Frage, welche der beiden reproduktionsmedizinischen Technologien aus ethischer Sicht vorzugswürdig wäre. Allerdings ist dieser Vergleich vielleicht obsolet. Denn ein reproduktionsmedizinischer Einsatz der Genomeditierung ohne nachfolgende Präimplantationsdiagnostik und Embryonenselektion scheint aus Gründen der Sicherheit ohnehin nicht in Betracht zu kommen (National Academy of Sciences et al., 2020). Insofern man davon ausgeht, dass der moralische Status des Embryos es verbietet, im Rahmen assistierter Reproduktionsmedizin eine Selektion von Embryonen vorzunehmen, wären somit sowohl PID als auch Genomeditierung nicht akzeptabel.

\subsection{Zusammenfassung und Ausblick}

Zusammenfassend kann festgehalten werden, dass sowohl im Diskurs zur Epigenetik als auch zur Genomeditierung ethische Argumente mit Bezug auf zukünftige Generationen und Gerechtigkeit eine zentrale Rolle spielen.

$\mathrm{Zu}$ Beginn des vorliegenden Kapitels wurden die Annahmen des genetischen Determinismus, der Grundlage eines genetischen Essentialismus ist, des epigenetischen Determinismus sowie des genetischen und epigenetischen Exzeptionalismus analysiert und kritisch diskutiert. Die Ausführungen zum ethischen Diskurs zur Epigenetik zeigen, dass die Vorstellung eines epigenetischen Determinismus nicht nur im populärwissenschaftlichen, sondern auch im ethischen Diskurs mitunter auszumachen ist. Ethische Debatten zur Epigenetik distanzieren sich aber z. T. von diesem deterministischen Verständnis. Das hat zur Folge, dass sich der Fokus im ethischen Diskurs zur Epigenetik von Verantwor-

17 Diese Argumentation geht auf Habermas (2001) zurück. Sie findet sich innerhalb der Debatte zum Vergleich von Genomeditierung und Präimplantationsdiagnostik bei Rehmann-Sutter (2018). Vgl. auch Lanzerath (2018: 121). 
tung für die eigene Gesundheit und die nachfolgender Generationen auf Gerechtigkeit verschiebt. Gemeint ist hier Gerechtigkeit z. B. in Bezug auf Zugang zu gesunden Umweltbedingungen, unabhängig davon, ob diese mit oder ohne Vermittlung über das Epigenom zur Gesundheit beitragen.

Aus einer Analyse des Diskurses zur Genomeditierung wird ersichtlich, dass v. a. Keimbahneingriffe ethisch untersucht werden und hierbei eine Konzentration auf den Aspekt der Vererbung stattfindet. Es ist zu fragen, ob damit ein impliziter genetischer Determinismus oder sogar ein genetischer Essentialismus einhergeht: Der Determinismus könnte hierbei in der Zentralität des Aspekts der Vererbbarkeit liegen, da nur genetische Information vererbt wird. Spielt der Aspekt der Vererbung und der Veränderung des Genoms in Debatten zur Genomeditierung eine ausschlaggebendere Rolle als die Sicherheitsproblematik? Wird dem Problem, dass Embryonen und deren potenzielle Nachkommen in einen Keimbahneingriff nicht einwilligen können, deshalb ein so hoher Stellenwert zugeschrieben, da es sich um einen genetischen Eingriff handelt?

Unter einer solchen Voraussetzung eines starken genetischen Determinismus, der um einen epigenetischen Determinismus ergänzt würde, wäre nicht nur die Genomeditierung, sondern auch die Epigenomeditierung gerade deshalb ethisch relevant, da auch sie einen Einfluss auf das Genom hätte. Wie dieser Einfluss von Genomeditierung und Epigenomeditierung jeweils ethisch bewertet wird, hängt daher zunächst davon ab, ob die Annahme eines genetischen und epigenetischen Determinismus vertreten wird. Diese Annahmen werden im ethischen Diskurs zunehmend kritisch gesehen, da sie aus naturwissenschaftlicher Sicht nicht bestätigt werden können. Insbesondere in populärwissenschaftlichen Debatten scheinen sie hingegen fortzubestehen, was schließlich auch Einfluss auf die öffentliche Diskussion nimmt. Da gerade zur Genomeditierung eine breite öffentliche Diskussion erforderlich ist, ist ein reflektierter Umgang mit den verschiedenen -ismen, die hier analysiert wurden, zentral.

\subsection{Literaturverzeichnis}

Agius, E./Busuttil, S. (Hrsg.) (1998): Germ-line intervention and our responsibilities to future generations. Springer/Kluwer, Dordrecht.

Birnbacher, D. (2018): Gentechnisches Enhancement. In: Hucho, F. et al. (Hrsg.): Vierter Gentechnologiebericht. Bilanzierung einer Hochtechnologie. Nomos, Baden-Baden: 237-250.

Bode, P. (2016): Identität und Nichtidentität. Intergenerationale Gerechtigkeit als Gegenstand einer Ethik der Epigenetik. In: Heil, R. et al. (Hrsg.): Epigenetik. Ethische, rechtliche und soziale Aspekte. Springer, Wiesbaden: 59-73. 
Brokowski, C. (2018): Do CRISPR germline ethics statements cut it? In: The CRISPR Journal 1(2): 115125. DOI: $10.1089 /$ crispr.2017.0024.

Cavaliere, G. (2018): Genome editing and assisted reproduction. Curing embryos, society or prospective parents? In: Med Health Care and Philos 21(2): 215-225. DOI: 10.1007/s11019-017-9793-y.

Dar-Nimrod, I./Heine, S. J. (2011): Genetic essentialism. On the deceptive determinism of DNA. In: Philosophical Bulletin 137(5): 800-818. DOI: 10.1037/a0021860.

Dupras, C./Bunnik, E. M. (2021): Toward a framework for assessing privacy risks in multi-omic research and databases. In: Am J Bioeth, Online-Publikation 12.01.2021. DOI: 10.1080/15265161.2020.1863516.

Dupras, C./Ravitsky, V. (2016): The ambiguous nature of epigenetic responsibility. In: J Med Ethics 42(8): 534-541. DOI: 0.1136/medethics-2015-103295.

Dupras, C. et al. (2014): Epigenetics and the environment in bioethics. In: Bioethics 28(7): 327-334. DOI: 10.1111/j.1467-8519.2012.02007.x.

Dupras, C. et al. (2018): Epigenetic discrimination. Emerging applications of epigenetics pointing to the limitations of policies against genetic discrimination. In: Frontiers in Genetics 9: 202, OnlinePublikation 08.06.2018. DOI: 10.3389/fgene.2018.00202.

Dupras, C. et al. (2020): Selling direct-to-consumer epigenetic tests. Are we ready? In: Nat Rev Genet 21: 335-336. DOI: 10.1038/s41576-020-0215-2.

Falomir, S. (2018): Epigenetics and metaphor. Language of limits. In: Technoethic Arts 16(3): 259-302. DOI: 10.1386/tear.16.3.295_1.

Garrison, N. A. et al. (2019): Genomic contextualism. Shifting the rhetoric of genetic exceptionalism. In: Am J Bioeth 19(1): 51-63. DOI: 10.1080/15265161.2018.1544304.

Green, M. J./Botkin, J. R. (2003): „Genetic Exceptionalism“ in medicine. Clarifying the differences between genetic and nongenetic tests. In: Ann Intern Med 138(7): 571-575. DOI: 10.7326/0003-4819138-7-200304010-00013.

Guttinger, S. (2020): Editing the reactive genome. Towards a postgenomic ethics of germline editing. In: J Appl Philos 37(1): 58-72. DOI: 10.1111/japp.12367.

Habermas, J. (2001): Die Zukunft der menschlichen Natur. Auf dem Weg zu einer liberalen Eugenik? Suhrkamp, Frankfurt am Main.

Hanna, C. W./Kelsey, G. (2014): The specification of imprints in mammals. In: Heredity 113: 176-183. DOI: $10.1038 /$ hdy.2014.54.

Holm, S. (2019): Let us assume that gene editing is safe. The role of safety arguments in the gene editing debate. In: CQ 28(1): 100-111. DOI: 10.1017/S0963180118000439.

Huang, J. Y./King, N. B. (2018): Epigenetics changes nothing. What a new scientific field does and does not mean for ethics and social justice. In: Public Health Ethics 11(11): 69-81. DOI: 10.1093/phe/ phx013.

Lanzerath, D. (2018): Ethische Kriterien und Argumente im Wandel der Zeit. In: Hucho, F. et al. (Hrsg.): Vierter Gentechnologiebericht. Bilanzierung einer Hochtechnologie. Nomos, Baden-Baden: 103128. 
Lewens, T. (2020): Blurring the germline. Genome editing and transgenerational epigenetic inheritance. In: Bioethics 34(1): 7-15. DOI: 10.1111/bioe.12606.

Mintz, R. L. et al. (2019): Will CRISPR germline engineering close the door to an open future? In: Sci Eng Ethics 25(5): 1409-1423. DOI: 10.1007/s11948-018-0069-6.

National Academy of Sciences et al. (2020): Heritable human genome editing. Consensus study report. The National Academies Press, Washington.

Nelkin, D./Lindee, M. (1995): The DNA mystique. The gene as a cultural icon. W. H. Freeman and Company, New York.

Piotrowska, M. (2019): Why is an egg donor a genetic parent, but not a mitochondrial donor? In: CQ 28(3): 488-498. DOI: 10.1017/s0963180119000410.

Rehmann-Sutter, C. (2018): Why human germline editing is more problematic than selecting between embryos. Ethically considering intergenerational relationships. In: The New Bioethics 24(1): 9-25. DOI: 10.1080/20502877.2018.1441669.

Resnik, D. B./Vorhaus, D. B. (2006): Genetic modification and genetic determinism. In: Philos Ethics Humanit Med 1(9), Online-Publikation 26.06.2006. DOI: 10.1186/1747-5341-1-9.

Savulescu, J. et al. (2015): The moral imperative to continue gene editing research on human embryos. In: Protein Cell 6(7): 476-479. DOI 10.1007/s13238-015-0184-y.

Schuol, S. (2016): Widerlegt die Epigenetik den Gendeterminismus? Es kommt darauf an ... In: Heil, R. et al. (Hrsg.): Epigenetik. Ethische, rechtliche und soziale Aspekte. Springer, Wiesbaden: 45-58.

Schuol, S. (2017): Das regulierte Gen. Implikationen der Epigenetik für Biophilosophie und Bioethik. Karl Alber, Freiburg/München.

Tappeser, B./Hoffmann, A.-K. (2004): Das überholte Paradigma der Gentechnik. Zum zentralen Dogma der Molekularbiologie fünfzig Jahre nach der Entdeckung der DNA-Struktur. In: AgrarBündnis e.V. (Hrsg.): Der kritische Agrarbericht 2004. ABL Verlag, Hamm: 220-224. Unter: https://www.kritischer-agrarbericht.de/fileadmin/Daten-KAB/KAB-2004/Tappeser_Hoffmann. pdf [11.02.2021].

Tobi, E. W. et al. (2014): DNA methylation signatures link prenatal famine exposure to growth and metabolism. In: Nat Commun 5(5592), Online-Publikation 26.11.2014. DOI: 10.1038/ncomms6592.

van Dijke, I. et al. (2018): The ethics of clinical applications of germline genome modification. A systematic review of reasons. In: Hum Reprod 33(9): 1777-1796. DOI: 10.1093/humrep/dey257.

Waggoner, M. R./Uller, T. (2015): Epigenetic determinism in science and society. In: New Genetics and Society 34(2): 177-195. DOI: 10.1080/14636778.2015.1033052.

Walter, J./Hümpel, A. (Hrsg.) (2017): Epigenetik. Implikationen für die Lebens und Geisteswissenschaften. Nomos, Baden-Baden. 